\title{
Integrated Operation of Diagnostic and Control Systems
}

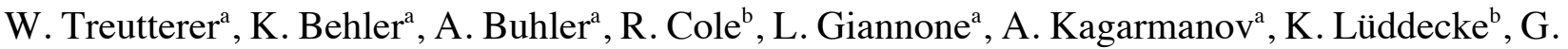 \\ $\mathrm{Neu}^{\mathrm{a}}$, G. Raupp ${ }^{\mathrm{a}}$, M.Reich ${ }^{\mathrm{a}}$, D. Zasche ${ }^{\mathrm{a}}$, T. Zehetbauer ${ }^{\mathrm{a}}$ and ASDEX Upgrade Team ${ }^{\mathrm{a}}$ \\ ${ }^{a}$ Max-Planck Institut für Plasmaphysik, Garching, Germany, EURATOM Association \\ ${ }^{b}$ Unlimited Computer Systems GmbH, Iffeldorf, Germany
}

\begin{abstract}
In fusion research the ability to generate and sustain high performance fusion plasmas gains more and more importance. Optimal combinations of magnetic shape, temperature and density profiles as well as the confinement time are identified as advanced regimes. Safe operation in such regimes will be crucial for the success of ITER and later fusion reactors. The operational space, on the other hand, is characterized by nonlinear dependencies between plasma parameters. Various MHD limits must be avoided in order to minimize the risk of a disruption.

Sophisticated feedback control schemes help to tackle this challenge. But these in turn require detailed information on plasma state in time to allow proper reaction. Control system and diagnostic systems therefore must establish a symbiotic relationship to carry out such schemes. Today, all major fusion devices implement such a concept.

An implementation of such a concept with sustained integration is presented using the example of ASDEX Upgrade. It covers data communication via a real-time network, synchronization mechanisms for data-driven algorithm execution as well as operational aspects and exception handling for failure detection and recovery. A modular distributed software framework offers standardized user algorithm interfaces, automated workflow procedures and the application of various computer and network hardware components. Designed with a special focus on reliability, robustness and flexibility, it is a sound base for exploring ITER-relevant plasma regimes and control strategies.
\end{abstract}

Keywords: advanced scenario, event handling, feedback control, real-time diagnostic, service oriented architecture, system integration

\section{Introduction}

In the past decade much effort in thermonuclear fusion research has been spent on investigating methods to substantially enhance plasma performance. The aim is to establish reproducible conditions allowing ITER, the next international tokamak experiment, to produce selfheated plasmas close to steady-state operation [1], and to prove that a future fusion reactor can be operated in an economic way.

\subsection{A path to high performance plasmas}

Plasma performance in this respect can be defined in terms of proper combinations of major plasma parameters. Plasma density, temperature and the thermal insulation given by the energy confinement time $\tau_{\mathrm{E}}$ form the fusion triple product $n \cdot T \cdot \tau_{\mathrm{E}}$, a measure for the efficiency of the fusion process.

The alleged simplicity, however, hides complex interrelations between the factors. In fact these scalar numbers are representations of spatial distributions or profiles. Complex physics - from magnetohydrodynamics over particle diffusion and transport to radiation of energy and plasma-wall interaction governs the evolution of these profiles. Behind the three factors lies a great number of internal variables (or states), which are not all directly accessible via the small number of actuating systems, available in a fusion reactor. Even more, plasma physics is highly nonlinear and can be linearized only within distinct operation regions or scenarios with characteristic behavior, often separated by sharp boundaries.

H-mode and advanced scenarios with internal transport barriers or hybrid scenarios are prominent examples of such optimized and stable regimes. Such advanced scenarios provide high beta value and high bootstrap fraction, both important for the economical use of input power and steady-state operation and thus for ITER [2]. However, beyond their boundaries, magnetohydrodynamic instabilities, such as ideal kink limit, resistive wall modes (RWM) or neoclassical tearing modes (NTM), can lead to abrupt losses of stored energy and to plasma disruption [3] [4].

As a consequence, the individual factors of the fusion product cannot be increased independently and the temporal evolution plays an important role. There is no simple recipe to optimize the fusion product. On the contrary, the path to high performance is formed by a progression of scenarios, and an important research focus is to find transition paths with safe margins to detrimental limits.

\subsection{Plasma control with feedback}

Feedback control is an indispensable tool for this venture. Given the evolution path through the scenarios as a reference and plasma state as feedback, it can keep excursions small. Automated reaction rules serve for accurate tracking and thus for sufficient safety margin to the limits. They compensate deficiencies of incomplete 


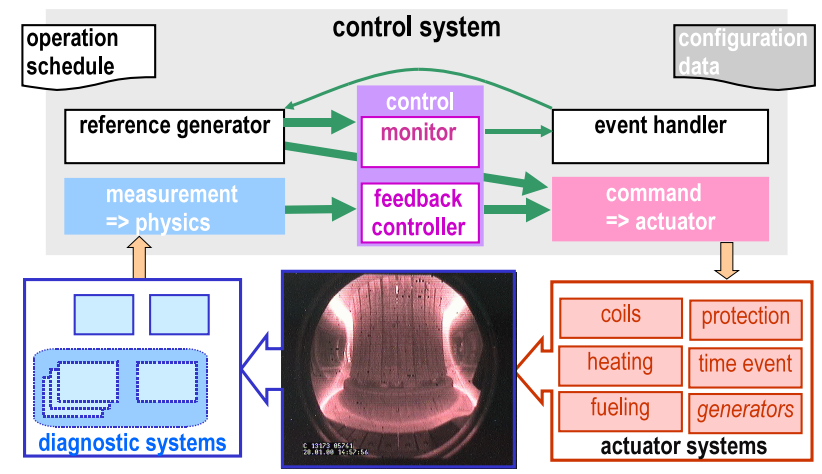

Fig. 1: Basic plasma control scheme

physics prediction models, unforeseen events or measurement noise. For tokamak fusion devices feedback controllers have been intrinsic components, at least, since diverted geometries with elongated plasma shapes boosted energy confinement but simultaneously introduced vertical position instability [5]. Meanwhile, standard tokamak operation is assisted by many more feedback control loops from single-input single-output (SISO) plasma current or density control to multivariable (MIMO) plasma shape or current profile control.

Owing to the complexity of the task, feedback control is not only performed in its classical continuous data processing way such as PID, state-space or relay regulators. These are complemented by asynchronous reactions on pre-defined conditions, also known as event handlers. Thus, apart from avoiding critical regions it is even possible to stabilize and remove MHD modes or at least to mitigate their effects [6].

From its definition, feedback control involves measurement or reconstruction of the controlled variables. In fusion devices this process often makes use of dedicated physical effects with considerable complexity and is conveniently implemented by a specialized diagnostic system. While most of the elaborate reconstruction algorithms are run a posteriori after a plasma discharge, key diagnostic systems such as magnetic flux and field measurement always have supplied their relevant signals to the feedback controllers in real-time. Fig. 1 shows the principal scheme of a plasma control loop from measurement and evaluation over feedback controllers, monitors and event handlers to actuator systems and, finally, the plasma to be controlled.

\subsection{Integration with real-time diagnostics}

Originally, integration of the diagnostic systems with the control system was kept to a minimum. In many cases the control system captured the diagnostic's analogue outputs of pre-amplified sensor probes. Subsequently, the control system fed them into a simplified real-time version of the diagnostic's offline reconstruction. Synchronizing trigger events distributed with low latency in real-time provided coherent operation. ASDEX Upgrade's on-line equilibrium reconstruction is an example for this technique [7]. Other approaches defined pairs made up of a diagnostic and a dedicated feedback controller as subsystems of plasma control. In JET vertical position control and the digitizers of the magnetic diagnostic form a selfcontained entity, just loosely connected to the outside via JET's Asynchronous Transfer Mode (ATM) network [8].

Albeit the low degree of integration offers advantages in the independence of operation, the redundancy of evaluated results and the variability in component design, such simple operation models are no longer sufficient in view of the challenges of advanced scenario control. The more sophisticated control and event handling methods, the better the information about plasma state must become - both in quantity and quality.

Often the required algorithms already exist in form of off-line codes, executed after a discharge - and in knowledge of the full plasma state. Progress in computer technology with fast clock rates of more than $3 \mathrm{GHz}$ as well as parallel multi-threaded execution on multiple processor cores meanwhile permits running such algorithms during a plasma pulse, fast enough that feedback control can use the results as input. Diagnostic systems with this capability are commonly attributed as real-time diagnostics. Modern plasma control is going to need a lot of them.

Reconstruction algorithms, however, are subject to permanent modification as the underlying physics model improves. A 'hard-wired' interface between real-time diagnostic and control system like analogue electronic voltages would become outdated in short time. On the other hand these high-level algorithms often depend on plasma state information from other diagnostics, enforcing a bi-directional data flow for input and for output data. Furthermore, some of the algorithms are valid only during some phases, where dedicated boundary conditions are met. A similar problem arises, when a sensor failure is encountered. Then subsequent users must be made aware of the state of the results. These examples illustrate that the integration between diagnostic and control systems must become much tighter than it used to be.

\subsection{Integration requirements}

What is needed is a standardized hardware and software interface between the systems, which is flexible enough to support a large variety of attached systems and to survive many system evolution cycles. What is needed is an operation model covering the cases, where produced data are unavailable or invalid. What is needed is a synchronization method guaranteeing the consistency of data coming from different partners with individual sampling rates and trigger times. What is needed is a sustained solution, which is open for extension and not tailored to a temporarily actual use case. Last but most important, plasma control as well as real-time diagnostics must evolve from a conglomeration of separate components like shape control or temperature profile reconstruction to an integrated application accounting for their context defined by high performance plasma physics. Efforts in this direction are taken in all of the major fusion devices. 


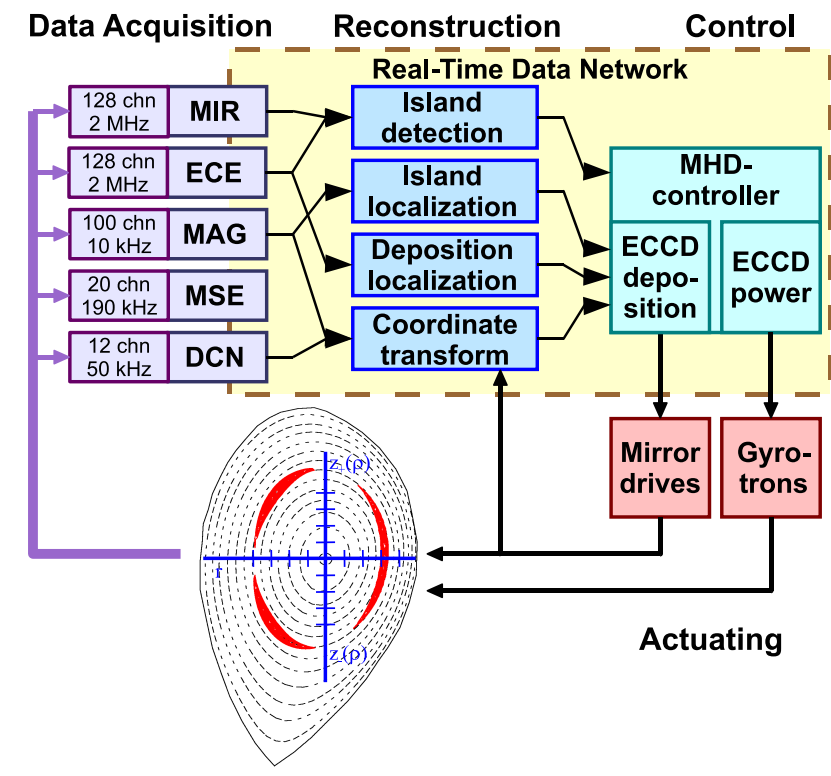

Fig. 2: ASDEX Upgrade NTM control scheme

In the following sections the main concepts for an integrated operation of diagnostic and control systems are presented. Section 2 introduces an example project from ASDEX Upgrade - NTM control with ECCD and steerable mirrors, which will be used to illustrate some details. Section 3 shows, how diagnostic systems and control system can jointly be operated and deals with complexity management, user interface and common workflow. In section 4 the real-time processing aspects of integration, real-time networks, latency restrictions, data quality management and event handling support will be discussed. A survey of integrated systems in present tokamak facilities will be shown in section 5 . Finally, section 6 will summarize the topic with a brief outlook about future development in this area.

\section{An example: NTM control with ECCD}

Neoclassical tearing modes frequently occur in high beta scenarios, driven by low frequency saw teeth or fast particle excitation. They flatten the pressure profile over a magnetic island, lead to a hole in the bootstrap current profile and thus substantially deteriorate plasma confinement [6]. Electron Cyclotron Current Drive (ECCD) can be used to replace the missing current and to suppress the mode - if the gyrotron beam power is deposited at the appropriate location where the island resides. The task of the feedback control loop is to detect the island and direct the ECCD beam(s) such that the resonant flux surface coincides with the corresponding flux surface of the island.

There are a couple of approaches, which differ in the way the ECCD beams are directed to the correct location. DIII-D uses an array of 6 gyrotron beams aligned side-by-side and powers the appropriate gyrotron when a mode appears. While this method has a very fast response, it works only, as long as the island location resides in the span formed by the beam lines [9]. ASDEX Upgrade has four independently controllable gyrotrons with steerable mirrors [10]. The core problem, however, is in common for all approaches: to detect the island and the beam deposition location, information from various diagnostic systems must be combined in real-time and made accessible to the control system. Fig. 2 shows the logical structure of the ASDEX Upgrade control loop. The electron cyclotron emission radiometry (ECE) diagnostic measuring the electron temperature profile can detect both locations in terms of channels with distinct resonant frequencies. But in order to correlate them with modes or gyrotron beams and to transform the result to gyrotron mirror coordinates, information about the structure of the magnetic flux surfaces, the ECCD beam power value and the density profile which deflects the beam must be obtained from the Mirnov diagnostic (MIR), the magnetic diagnostic (MAG), the motional stark effect (MSE) diagnostic, the ECCD actuator system and from laser interferometry (DCN). Using the knowledge that NTMs only occur on rational flux surfaces an alternate reconstruction of the mode location without ECE data is available, too. A high-level diagnostic evaluation code (TORBEAM) to estimate the ECCD power deposition location in poloidal flux coordinates has been re-written and optimized for real-time operation [11]. A real-time network for sharing data connects all diagnostic systems and the control system [12].

This fairly complex scheme is typical for high performance control tasks. Therefore, the example is used for illustration throughout the following sections.

\section{Operation model}

The first lesson that can be learned from the example is that the real-time diagnostics involved nevertheless are not exclusively assigned to this control loop. The magnetic structure, for instance, is of interest for many other control purposes like saw tooth control, current or pressure profile reconstruction or disruption predictors. Hence, a rigid architecture oriented at the control loop is not adequate. The real-time diagnostic systems, as well as the control system components, must rather be treated as information services, publishing their results to any application requiring them. This approach is also in line with modern fusion control system framework design using generic application modules (GAMs) [13] and application versus infrastructure separation [14].

\subsection{Service oriented architecture}

Opening the view to generic service oriented architecture [15] [16] might look like an overkill on the first glance. It turns out, however, that it offers a compelling solution to manage the continuously growing complexity. Coming from the business world and being popular in web applications the service orientation introduces a number of beneficial principles. One of these is encapsulation, which decouples data producers and consumers into services and is the base for extensible and scalable system architectures. A central flow manager coordinates service execution (orchestration). Alternatively the services can self- 


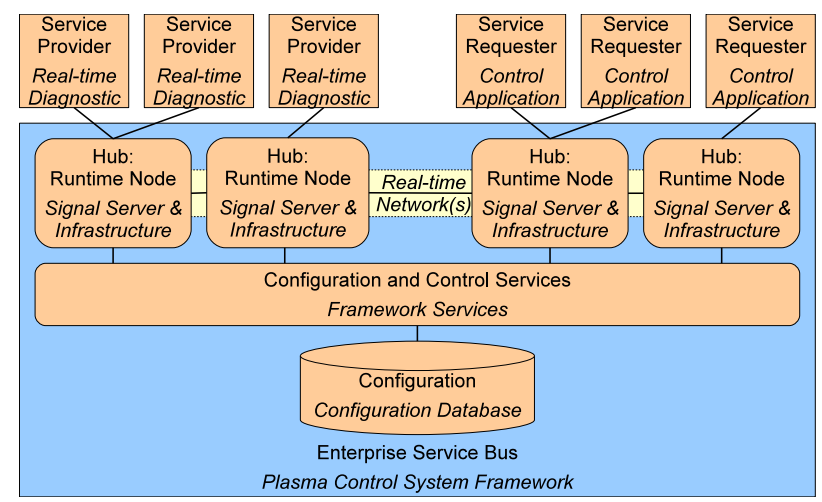

Fig. 3: Service Oriented Architecture (from [17]) compared to integrated control system architecture (in italics)

organize collaboration driven by events (choreography). On this foundation, service registration and lookup allow the flexible composition of control loops from several diagnostic and control services. The resulting structure can be easily applied to integrated plasma control, as Fig. 3 illustrates.

Another useful principle is the service description, defining configuration parameters as well as input and output characteristics of applications in a standardized format. From these descriptions data communication maps transparent to the diagnostic and control algorithms can be created automatically. This opens the possibility of restructuring control loop deployment and adapting it to the available computing equipment. Last but not least, a common communication protocol ensures that all components 'speak the same language'.

In the NTM example this philosophy allowed to integrate heterogeneous systems like the LabView $\mathrm{RT}^{\circledR}$ based magnetic diagnostic [18] and others built on a dedicated standard real-time diagnostic framework [19].

\subsection{Common workflow}

With service orientation the external interfaces of diagnostic and control systems become abstract. Collaboration via such generic interfaces requires a common workflow. The availability of all required systems can be monitored and operation can be made more reliable when they have to respond to the commands of a central state machine. Visualization of the overall system state helps the engineers and physicists in charge to operate the experiment.

The first common state of such a workflow is the preparation phase. It has to guarantee the consistency of collectively used configuration data. In the NTM example the ECE channel-to-frequency mapping is a constant parameter, which, however, can change between discharges, depending on the selected toroidal field. This mapping is also of importance for the TORBEAM projection. The common workflow defines that configuration settings are frozen at a certain point of time for the duration of the following discharge. Thus, ECE and TORBEAM always use the same mapping.

Subsequent phases deal with component initialization including infrastructure elements like networks. They are followed by the actual experiment phase consisting of technical plant systems preparation, plasma discharge and shutdown. Details on how to handle this part of the workflow are discussed in section 4 .

In the termination phase all cached experiment data are archived and each system is reset to a known initial state. The workflow also enforces clean-up sequences after failed or interrupted state transitions.

In summary the service oriented concept combines loose coupling of process implementations with a tight integration framework based on standardized interfaces and a centrally managed workflow.

\section{Real-time processing}

The experiment phase is the place where the productive work of the integrated system is done. One control system requirement dominates the design: the real-time capability. The meaning is simple - the overall reaction time of the whole processing chain from the sensor over the controller to the actuator must be fast enough to reproducibly yield a desired response. In technical terms, they must be considerably faster than the physical timescale of the most dynamic process to control. As a rule of thumb, a factor of 10 is appropriate in most cases.

\subsection{Real-time requirements}

The consequences are restrictive: execution times as well as data transport latencies must stay below guaranteed values, and data communication overhead must be minimized.

From the first demand it follows that time-consuming iterating approximations or recursive algorithms, although common in diagnostic evaluation codes, are prohibited as much as network protocols with congestion control like TCP/IP.

The second point calls for efficient network support of small data entities as produced by the majority of control system components. Analysis of typical network traffic shows small average data rates but high peak loads. Given the performance limitations of todays lowlatency networks it also suggests that only preprocessed physically meaningful quantities instead of bulk raw measurement data should be distributed by diagnostic systems. The 'real-time' prefix for networks and diagnostic systems indicates that they are designed and capable to fulfill these requirements.

\subsection{Data flow and quality management}

Another implication of the afore-mentioned real-time constraints is, that the system topology - the network connections and the data routing - is static during realtime operation. Otherwise, deterministic behavior cannot be ensured.

This raises the question, how systems should behave in situations when they cannot execute their algorithms and produce meaningful results. While some of the control system components operate continuously, 
diagnostic systems may be able to deliver data only within certain time windows. The NTM example holds a variety of cases. Laser interferometry can deliver plasma density measurements only after plasma breakdown. ECE microwaves can get cut-off at high densities inhibiting the calculation of electron temperature profiles. The MSE diagnostic can observe the motional stark effect only when the referenced neutral beam is fired. Conventional diagnostic systems in such cases just wait for a trigger from the timing system and capture data for a fixed time window. The filtering of valid data is postponed to the off-line evaluation. Unfortunately, this strategy is not applicable for real-time operation. In the integrated environment chains of client applications are waiting for input.

One possibility is to produce and distribute data only while certain operation conditions are met. Similarly clients would stall their operation, until input data become available. Such synchronization can be accomplished with blocking mechanisms as provided by real-time operation systems.

This method is not appropriate in all places. Event handling processes, for example, must detect missing or

Timestamp

- control cycle number or absolute time

- process synchronisation

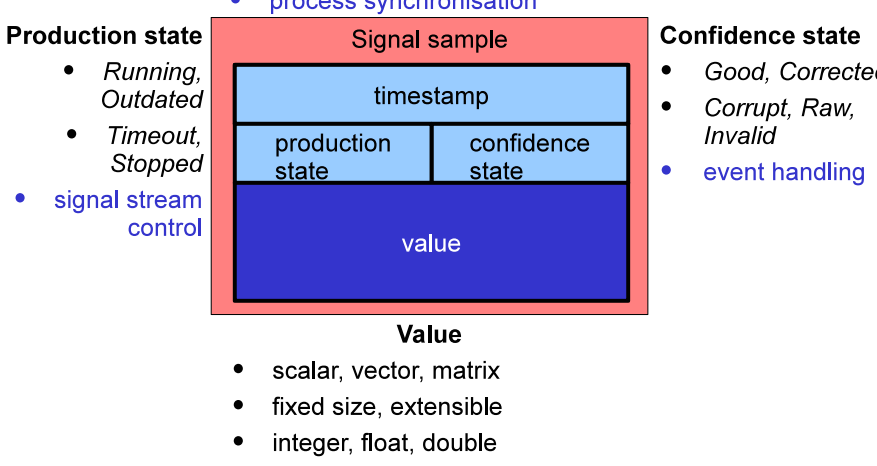

Fig. 4: ASDEX Upgrade Sample Definition

faulty data and react, instead of pausing. They require an indicator for data quality issued along with the payload data. Simple implementations use reserved values like zero or 'not a number' for this purpose. A versatile and safe implementation, however, would define separate quality tags with staged levels attached to each data sample. An example sample layout might look like in Fig. 4. Consequently used also in evaluation and control applications, such tags can build the foundation of an event handling strategy. In the NTM example they enable the NTM controller to select between the island locations calculated with ECE measurements and the redundant one based on magnetic reconstruction of the rational flux surfaces, if ECE should be in cut-off.

In the end both approaches have their justifications. But combining them can yield an extremely powerful system architecture.

\subsection{Data consistency and synchronization}

A last important challenge for integrated real-time operation of diagnostics and control is the consistency of data from different sources. Each diagnostic system as well as the control system may have its own sampling frequency and its individual processing time window and processing rate. In addition, processing delays may vary dependent on the complexity of the codes. Algorithms, on the other hand, often assume time-aligned input data with bounded frequency spectrum. If sample data are not consistent in this respect, aliasing and artifacts can result. Frequently these effects occur when data rates are similar but not equal. Still, however this aspect is often ignored and the latest sample value is used unconditionally. A minimum remedy is to time-stamp the samples. Thus, compliant samples can be selected. For the future, however, more sophisticated approaches like clock and trigger alignment as well as interpolation and frequency-filtering techniques for time-stamped input data must be envisaged.

\section{Survey of present tokamak facilities}

A recent survey of plasma control systems of the JET, JT-60U, DIII-D, ASDEX Upgrade and Tore Supra tokamak devices shows to which extent the use of realtime diagnostic systems has meanwhile grown [20]. DIII-D, for example, features magnetic equilibrium, lineaveraged density, electron temperature, NTM and plasma confinement reconstruction in real-time. Tore Supra feeds data from magnetic sensors, infrared interferometers, bolometers, hard X-ray diagnostic and infrared thermography to the control system. All facilities have in common that the integration is accomplished via real-time networks: ATM in JET, reflective memory solutions in DIII-D, JT-60U, ASDEX Upgrade and Tore Supra and Ethernet with User Datagram Protocol (UDP) as an alternative network at ASDEX Upgrade. In most cases predefined data structures and the related memory locations or datagram addresses are employed for the communication interface. Time stamping or data quality tagging, however, are found only in the ASDEX Upgrade integration framework [21].

\section{Conclusion}

Integrated operation of diagnostics and control is no new topic. Using diagnostic measurements is immanent to any control purpose. The latest objectives in fusion plasma control, particularly advanced scenario and MHD mode control, however, require a much higher degree of collaboration and introduce an unprecedented amount of versatility but also complexity. This development has boosted design and implementation of generalized integration concepts, which is still an ongoing process.

On the hardware side computer networks optimized for real-time application have replaced analogue connections with signal voltages. On the software side 
design principles resembling those of service oriented architectures help to master the heterogeneity of diagnostic and control systems on the basis of encapsulation combined with standardized interfaces and communication protocols and a centrally coordinated workflow. Design philosophies of diagnostic and evaluation applications must be extended to deal with real-time requirements as well as with consistency assurance and quality assessment of data stream processing. Much room for future improvements can be found here. Another exciting topic, not covered in this paper, is the test and validation of such integrated formations. Here DIII-D plays a leading role integrating also simulation capabilities in the system [9].

Still evolution in this area is very active. But the foundation for a solution suitable also for ITER and later fusion power plants has been laid.

\section{References}

[1] IAEA, Summary of the ITER Final Design Report, ITER EDA Documentation Series No 22 (Vienna, 2001)

[2] A. C. C. Sips, Advanced scenarios for ITER operation, Plasma Phys. Control. Fusion 47 A19, 2005, doi:10.1088/0741-3335/47/5A/003

[3] J. R. Ferron, D. P. Brennan, T. A. Casper, A. M. Garofalo, C. M. Green- field, A. W. Hyatt, J. R. Jayakumar, L. C. Johnson, J. E. Kinsey, R. J. La Haye, L. L. Lao, E. A. Lazarus, J. Lohr, T. C. Luce, M. Murakami, M. Okabayashi, C. C. Petty, P. A. Politzer, R. Prater, H. Reimerdes, E. J. Strait, T. S. Taylor, A. D. Turnbull, J. G. Watkins, M. R. Wade, and W. P. West, "Progress toward sustained high-performance advanced tokamak discharges in DIII-D," Proceedings of the 29th Plasma Physics and Con- trolled Fusion Conference, Montreux, Switzerland European Physical Society, Paris, France, 2002 , Vol. 26B, p. 1.060 .

[4] H. Zohm. et al., "MHD limits to tokamak operation and their control", Plasma Phys. Control. Fusion 45 (2003) A163, doi: 10.1088/0741-3335/45/12A/012

[5] S.C. Jardin, D.A. Larrabee, Feedback stabilization of rigid axisymmetric modes in tokamaks. Nuclear Fusion 22 (1982) 1095-1098.

[6] M. Maraschek et al, Active control of MHD instabilities by ECCD in ASDEX Upgrade, Nuclear Fusion 45 (2005) 1369.

[7] P.J. Mc Carthy, O. Kardaun, H. Bruhns, K. Lackner, Optimal selection of plasma parameters and magnetic measurement locations for tokamak feedback control, Fusion Technology (1989), 687-693.

[8] F. Sartori et al, The JET PCU project: An international plasma control project, Fusion Engineering and Design, 83 (2008), 202-206, doi:10.1016/j.fusengdes.2007.10.013.

[9] D. A. Humphreys, J. R. Ferron, R. J. La Haye, T. C. Luce, C. C. Petty, R. Prater, and A. S. Welander, Active control for stabilization of neoclassical tearing modes, Physics of Plasmas 13, 056113 (2006)

[10] A. Manini et al, Development of a Feedback System to Control MHD Instabilities in ASDEX Upgrade, Fusion
Engineering and Design 82 (2007), 995-1001, doi:10.1016/j.fusengdes.2007.07.031

[11] M. Reich et al, ECCD-based NTM control using the ASDEX Upgrade real-time system, (submitted to EPS 2010)

[12] W. Treutterer et al, Real-time signal communication between diagnostic and control in ASDEX Upgrade, Fusion Engineering and Design, 85 (2010), 466-469, doi:10.1016/j.fusengdes.2010.04.031

[13] A. Neto et al, MARTe: a Multi-Platform Real-Time Framework", 16th IEEE NPSS Real Time Conference, May 10-15, 2009, Beijing, proc. to be published on IEEE Transactions on Nuclear Science (TNS)

[14] G. Raupp, et al, Control process structure of ASDEX Upgrade's new control and data acquisition system, Fusion Eng. Des. 74 (2005) 697-705.

[15] M. Bell, Introduction to Service-Oriented Modeling. Wiley \& Sons. (2008), pp. 3. ISBN 978-0-470-14111-3

[16] M. Endrei et al, Patterns: Service-oriented Architecture and Web Services, IBM redbooks SG24-6303-00 (2004), p. 25

[17] M. Keen et al, Patterns: Implementing an SOA Using an Enterprise Service Bus, IBM redbooks SG24-6346-00 (2004), p79

[18] L. Giannone, Data acquisition and real-time signal processing of plasma diagnostics on ASDEX Upgrade using LabVIEW RT, Fusion Engineering and Design 85, 34 (2010), 303-307, doi:10.1016/j.fusengdes.2010.03.030

[19] M. Reich et al, Real-time diagnostics and their applications, (to be published in Fusion Science and Technology)

[20] K. Kurihara, J.B. Lister, D.A. Humphreysc, J.R. Ferron, W. Treutterer, F. Sartori, R. Feltone, S. Bremond, P. Moreau et al, Plasma control systems relevant to ITER and fusion power plants, Fusion Engineering and Design 83, 7-9 (2008), 959-970, doi:10.1016/j.fusengdes.2008.06.027

[21] W. Treutterer, et al., Real-time diagnostics at ASDEX Upgrade-Integration with MHD feedback control, Fusion Eng. Design (2007), doi:10.1016/j.fusengdes.2007.09.006 